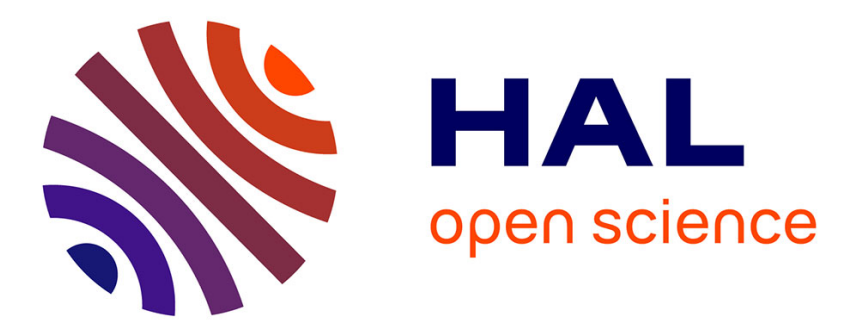

\title{
Predictive Control of Chained Systems: a Necessary Condition on the Control Horizon
}

\author{
Estelle Courtial, Matthieu Fruchard, Guillaume Allibert
}

\section{To cite this version:}

Estelle Courtial, Matthieu Fruchard, Guillaume Allibert. Predictive Control of Chained Systems: a Necessary Condition on the Control Horizon. International Conference on Robotics and Automation (ICRA12), May 2012, Saint Paul, United States. pp.109-114. hal-00716756

\section{HAL Id: hal-00716756 https://hal.science/hal-00716756}

Submitted on 11 Jul 2012

HAL is a multi-disciplinary open access archive for the deposit and dissemination of scientific research documents, whether they are published or not. The documents may come from teaching and research institutions in France or abroad, or from public or private research centers.
L'archive ouverte pluridisciplinaire HAL, est destinée au dépôt et à la diffusion de documents scientifiques de niveau recherche, publiés ou non, émanant des établissements d'enseignement et de recherche français ou étrangers, des laboratoires publics ou privés. 


\title{
Predictive Control of Chained Systems: a Necessary Condition on the Control Horizon
}

\author{
Estelle Courtial, Matthieu Fruchard, Guillaume Allibert
}

\begin{abstract}
This paper deals with state feedback control of chained systems based on a Nonlinear Model Predictive Control (NMPC) strategy. Chained systems can model many common nonholonomic vehicles. We establish a relation between the degree of nonholonomy and the minimum length of the control horizon so as to make the NMPC feasible. A necessary condition on the control horizon of NMPC is given and theoretically proved whatever the dimension of the chained system considered. This relation is used to design a NMPC-based control strategy for chained systems. One of the advantages of NMPC is the capability of taking into account the constraints on state and on control variables. The theoretical results are illustrated through simulations on a $(2,5)$ chained system, describing a car-like vehicle with one trailer. Difficult motion objectives that require a lateral displacement are considered.
\end{abstract}

\section{INTRODUCTION}

The interest in chained systems stems from the fact that the kinematic model of many common nonholonomic vehicles (unicycle, car, car-like vehicle with $n$ trailers, etc.) can be converted into this form [1]. The stabilization problem of nonholonomic vehicles has been largely investigated in the literature [2], [3]. Two control tasks can be distinguished: trajectory tracking and fixed point asymptotic stabilization. While the trajectory tracking is a rather simple problem (at least under the persistence of motion assumption), point-stabilization is far from straightforward when full-state stabilization (position and orientation) is considered. Albeit intrinsically controllable, a nonholonomic system has a linearized model which loses its controllability property at any fixed point. Therefore, linear control techniques cannot be used to stabilize this system at a given configuration. Moreover Brockett [4] showed that no continuous time-invariant feedback control could asymptotically stabilize this class of systems. Time-varying [5], [6], [7], discontinuous [8] and hybrid [9] feedbacks have been proposed. Recently, practical full-state stabilization has been addressed via the use of transverse functions [10]. This control approach uses additional virtual control inputs through a dynamic extension, providing practical stabilization along any (even non admissible) trajectory or fixed point with a priori bounded error.

E. Courtial is with the Laboratory PRISME, University of Orléans, 8 rue Léonard de Vinci, 45072 Orléans Cedex, France, estelle.courtialeuniv-orleans.fr

M. Fruchard is with the Laboratory PRISME, University of Orléans, 63 Av de Lattre de Tassigny, 18020 Bourges Cedex, France, matthieu.fruchard@univ-orleans.fr

G. Allibert is with the Laboratory I3S-CNRS, University of Nice-Sophia Antipolis, Bât Euclide B, Les Algorithmes, 2000 route des lucioles BP121, 06903 Sophia Antipolis Cedex, France, guillaume.alliberteunice.fr
Model Predictive Control implicitly provides a discontinuous feedback thereby bypassing Brockett's condition. Indeed, the NMPC strategy is based on the receding horizon principle and is formulated as solving on-line a nonlinear optimization problem, see [11] for a survey. The basic concepts of NMPC are the explicit use of a model to predict the process behavior over a finite prediction horizon $N_{p}$ and the minimization of a cost function with respect to a sequence of $N_{c}$ controls where $N_{c}$ is the control horizon. If NMPC is often applied to trajectory tracking of nonholonomic systems, only a limited number of studies deals with stabilization [12], [13], [14]. In [14], the authors studied the stability of unconstrained discrete-time systems controlled using NMPC. Under assumptions of detectability and boundedness of the cost function, a prediction horizon length was determined to ensure stability. In [12], the authors relaxed the restrictive terminal constraint by using a timevarying (exponential) weighted matrix to ensure stability. It was not sufficient to guarantee the full-state control from any initial configuration. In all the aforementioned papers, the control horizon was chosen equal to the prediction horizon. Nevertheless the control horizon plays a crucial role by providing a sequence of controls, similar to a manoeuvre, to drive the nonholonomic vehicle to its desired position. The question is to know how many controls are needed and therefore what is the minimal length of the control horizon $N_{c}$ sufficient to make the NMPC feasible?

The objective of this paper is to point out and illustrate how the control horizon can be appropriately chosen in order to guarantee the feasibility of the predictive control for chained systems. We establish a relationship between the degree of nonholonomy and the minimum length of the control horizon. This necessary condition of NMPC feasibility is theoretically proved whatever the dimension of the chained system considered.

The paper is organized as follows: section II is devoted to a brief recap of NMPC design, nonholonomic vehicles and chained systems. In section III, the problem is stated: how can we determine the minimal control horizon $N_{c}$ that will ensure the NMPC feasibility ? A necessary condition on $N_{c}$ is given for the state feedback control of chained systems. In section IV, the NMPC design is detailed. In section $\mathrm{V}$, simulations on a $(2,5)$ chained system, describing a car vehicle with one trailer, highlight the decisive role of the control horizon. Constraints on state and control variables are also taken into account. Conclusions are finally given. 


\section{BACKGROUND}

\section{A. $N M P C$}

The control objective is usually to steer the state to the origin or to an equilibrium state. A suitable change of coordinates transforms the second problem into the first which, therefore, we consider in the sequel. Consider the state $x$ at the time $k$, the cost function is defined by:

$$
J(x, u)=\sum_{j=k}^{k+N_{p}} L(x(j), u(j))+F\left(x\left(k+N_{p}\right)\right)
$$

where $L$ is a quadratic function and $\left.x\left(k+N_{p}\right)\right)$ is a terminal constraint added to ensure the stability of the closed-loop system. The classical NMPC formulation can be written as follows:

$$
\min _{\widetilde{u}} J(x, u) .
$$

The cost function $J$ is minimized over the prediction horizon $N_{p}$ with respect to the control sequence. The control sequence $\widetilde{u}=u(k), u(k+1), \ldots, u\left(k+N_{c}\right), \ldots, u\left(k+N_{p}-1\right)$ is composed of $N_{c}$ different controls where $N_{c}$ is the control horizon. From $u\left(k+N_{c}+1\right)$ to $u\left(k+N_{p}-1\right)$, the inputs are constant and equal to $u\left(k+N_{c}\right)$. Only the first element of the computed optimal sequence of controls is really applied to the process. At the next sampling instant, the prediction horizon moves one step forward and the whole procedure is repeated with the updated measurements.

The main advantage of NMPC is its ability to handle constraints. Constraints on states, inputs or outputs can easily and explicitly be added to the optimization problem (2).

\section{B. Modeling of nonholonomic vehicles}

This section recalls some properties of nonholonomic vehicles. For notions on Lie algebras and differential geometry, see [15], [16].

Wheeled robots are characterized by non completely integrable velocity constraints resulting from the rolling without slipping assumption $\left.<a_{i}(z), \dot{z}\right\rangle=0, i=1, \cdots, q$, where $z \in N$ is the configuration of the vehicle in an $n$ differentiable manifold $N$, and the $a_{i}$ 's are assumed smooth and independent [17]. Nonholonomic vehicles can hence be modeled by a kinematic driftless control system:

$$
\dot{z}=\sum_{i=1}^{m} u_{i} Z_{i}(z), \quad z(0)=z_{0}
$$

where $m=n-q<n, z_{0}$ is the initial configuration, the $u_{i}$ 's denote control variables and the $Z_{i}$ 's are smooth independent vector fields over $N$. The solution at time $t$ of (3) is denoted:

$$
z(t)=e^{t \sum_{i=1}^{m} u_{i} z_{i}}\left(z_{0}\right) .
$$

Let $\mathscr{Z}(N)$ be the Lie algebra of all vector fields on $N$ and $\mathfrak{g}=\mathscr{L}\left(Z_{1}, \cdots, Z_{m}\right)$ the Lie subalgebra of $\mathscr{Z}(N)$ generated by the vector fields $Z_{1}, \cdots, Z_{m}$. We assume that system (3) satisfies the Lie algebra rank condition on $N$ :

$$
\operatorname{dim}\left(\operatorname{span}\left\{Z(z): X \in \mathscr{L}\left(Z_{1}, \cdots, Z_{m}\right)\right\}\right)=n .
$$

Under assumption (5), Chow's theorem implies that system (3) is controllable [16].

In order to classify nonholonomic systems, we recall the concept of nonholonomic degree defined in [18].

Definition 1 Let $\mathfrak{g}_{1}=\operatorname{span}\left\{Z_{1}, \cdots, Z_{m}\right\}$ and recursively

$$
\mathfrak{g}_{k}=\mathfrak{g}_{k-1}+\left[\mathfrak{g}_{1}, \mathfrak{g}_{k-1}\right], \quad k \geq 2
$$

where $\left[\mathfrak{g}_{1}, \mathfrak{g}_{k-1}\right]=\operatorname{span}\left\{[X, Y]: X \in \mathfrak{g}_{1}, Y \in \mathfrak{g}_{k-1}\right\}$. Assuming the system is regular, we define the degree of nonholonomy as $p=\min \left\{i \in \mathbb{N}: \mathfrak{g}_{i}=\mathfrak{g}\right\}$.

The degree of nonholonomy is an image of the difficulty in controlling the system. Since the longer the Lie bracket is, the more difficult it is to move in its direction, the difficulty is the greatest when moving in the direction of the vector fields that belong to $\mathfrak{g} \backslash \mathfrak{g}_{p-1}$. This degree is an intrinsic property of the system, i.e. it does not depend on the coordinate system the control is written in.

Concerning the kinematic modeling of a wide class of wheeled robots, such as a unicycle with $n-3$ trailers, [1] gives a feedback change of coordinates $\left(z, u_{1}, u_{2}\right) \mapsto(x, v, w)$ converting system (3) in natural coordinates (i.e. defined on $S E(2) \times M$ with $M$ denoting a $(n-3)$-dimensional manifold) into a $(2, n)$ chained form, which is a particular case of system (3).

Definition $2 A(2, n)$ chained system is a kinematic system in the form (3) with two control inputs and the following vector fields:

$$
\left\{\begin{array}{l}
\dot{x}=X_{1}(x) v+X_{2} w, \quad x=\left(x_{1}, \cdots, x_{n}\right) \in \mathbb{R}^{n} \\
X_{1}=\left(1,0, x_{2}, \cdots, x_{n-1}\right) \\
X_{2}=(0,1,0, \cdots, 0)
\end{array}\right.
$$

The degree of nonholonomy of system (6) is $p=n-1$.

Example 1 Let us illustrate the modeling of a nonholonomic vehicle in a chained form. The car-like vehicle is commonly expressed in natural coordinates, i.e. it is modeled by system (3) with configuration $z=\left(x_{c}, y_{c}, \theta, \phi\right) \in S E(2) \times\left(-\frac{\pi}{2}, \frac{\pi}{2}\right)$, and vector fields given by:

$$
Z_{1}(z)=\left(\cos \theta, \sin \theta, \frac{\tan \phi}{l}, 0\right), \quad Z_{2}=(0,0,0,1) .
$$

$\left(x_{c}, y_{c}, \boldsymbol{\theta}\right)$ denotes the location of the rear wheels and orientation, and $\phi, l$ are respectively the front steering angle with respect to the car's body and the wheelbase. The kinematics of the car are given by system (3) with control vector fields (7) where $u_{1}, u_{2}$ are the linear and steering velocities of the vehicle. The car-like vehicle can be written in chained form (6) as:

$$
x=\left(\begin{array}{c}
x_{c} \\
\frac{\tan \phi}{l \cos ^{3} \theta} \\
\tan \theta \\
y_{c}
\end{array}\right),\left(\begin{array}{c}
v \\
w
\end{array}\right)=\left(\begin{array}{c}
u_{1} \cos \theta \\
\frac{u_{2}}{l \cos ^{3} \theta \cos ^{2} \phi}+3 \frac{\tan ^{2} \phi}{l^{2}} \frac{\sin \theta u_{1}}{\cos ^{4} \theta}
\end{array}\right) .
$$


We thus obtain the system (6) on the $(2,4)$ chained form, nilpotent of order 3 , defined on the Lie group $N=\mathbb{R}^{4}$, with vector fields:

$$
\begin{aligned}
& X_{1}(x)=\left(1,0, x_{2}, x_{3}\right) \\
& X_{2}=(0,1,0,0) .
\end{aligned}
$$

Note that the feedback (8) induces a singularity, since it is clear that defining the control input $w$ as well as $\tan \theta$ in the state $x$ requires that $\theta \in\left(-\frac{\pi}{2}, \frac{\pi}{2}\right)$.

\section{PROBLEM STATEMENT AND MAIN RESULT}

\section{A. Problem Statement}

Problem 1 Let $N_{c}$ denote the control horizon of a predictive control law and $(v, w) \in \mathbb{R}^{2}$, the control input of system (6). Consider a piecewise constant control family $\left\{v_{k}, w_{k}\right\}_{k \leq N_{c}}$ over $N_{c}$ sampling periods $T_{e}$. With notations of section II-B, let:

$$
s: \tilde{v} \mapsto x_{f}=e^{Y_{N_{c}}} \circ \cdots \circ e^{Y_{2}} \circ e^{Y_{1}} \circ x_{0}
$$

where $Y_{k}(x)=T_{e}\left(X_{1}(x) v_{k}+X_{2} w_{k}\right)$ and $\tilde{v}=(\tilde{v}, \tilde{w})$ is the control sequence with $\tilde{v}=\left(v_{1}, \cdots, v_{N_{c}}\right)$ and $\tilde{w}=\left(w_{1}, \cdots, w_{N_{c}}\right)$. What is the minimal control horizon $N_{c}$ such that there exists a control sequence $\tilde{v}$ solving equation (10) for any desired final configuration $x_{f}$, i.e. such that $s$ is surjective?

Solving Problem 1 is not always simple. Since the vector fields of system (6) are left-invariant ${ }^{1}$ on a Lie group, then equation (10) can be expressed as a product of exponentials, whose direct calculation is provided either using the Campbell-Hausdorff formula or using the group operation ${ }^{2}$ of the Lie group. For more generic systems in the form (3), producting the exponentials using the Campbell-Hausdorff formula will result in an infinite number of bracketings. If possible, it is thus preferable to feedback nilpotentize system (3) in order to avoid errors induced by the truncation of brackets longer than a given order [19]. Note however that feedback nilpotentization may induce singularities, thus limitating reachable points for a given control horizon.

\section{B. Solution to Problem 1 for chained systems}

Proposition 1 Solving Problem 1 for any $(2, n)$ chained system (6) generically requires a control horizon $N_{c}=p+1=n$.

The detailed proof is given in Appendix.

Remark 1 Proposition 1 gives a sufficient and necessary condition on $N_{c}$ for the wide class of nonholonomic vehicles modeled by the $(2, n)$ chained form, so as to ensure the existence of solutions to Problem 1 given arbitrary initial and final configurations.

Remark 2 In the scope of optimization-based control, there are slight differences with the formulation of Problem 1. Firstly, the goal in NMPC is not explicitly to regulate to zero

\footnotetext{
${ }^{1} \mathrm{~A}$ system is left-invariant if its vector fields are left-invariant, i.e. if $\mathrm{d} L_{\sigma}(\tau) \circ X(\tau)=X\left(L_{\sigma}(\tau)\right)$ with $L$ denoting the left-translation operator: $L_{\sigma}(\tau)=\sigma \tau$.

${ }^{2}$ Note that, in general, the group operation may be difficult to determine explicitly.
}

the error between the reference and the model output configuration obtained by applying $N_{c}$ control steps, but rather to minimize a weighted sum of errors over a prediction horizon $N_{p} \geq N_{c}$. Secondly, the handling of constraints either on the control inputs and/or on the states may reduce the set of solutions. Restrictions imposed using a NMPC approach thus result in relaxing the sufficient and necessary condition for solving the theoretical Problem 1 into a necessary condition, in practice, for solving the NMPC optimization problem formulated in the next section.

Let us discuss the case $N_{c}=p=n-1$, where $s$ is surjective for any motion along $\mathfrak{g}_{p-1} \backslash \mathfrak{g}_{p-2}$, but is no longer surjective on $\mathfrak{g} \backslash \mathfrak{g}_{p-1}$. This lack of solution is due to the nilpotentization, which is illustrated hereinafter by the significant example of the car in natural coordinates, and therefore should not be regarded as an intrinsic property of the system, but rather as a consequence of the choice of the coordinate system the nonholonomic system is written in.

Remark 3 Any translation along $\mathfrak{g} \backslash \mathfrak{g}_{2}$, corresponding to a pure transversal motion (along $X_{4}=\left[X_{1},\left[X_{1}, X_{2}\right]\right]=$ $(0,0,0,1))$, is clearly feasible by a car-like vehicle modeled by system (3) with vector fields (7) and $N_{c}=p=3$, but it requires that the orientation reach values forbidden by the nilpotentization (8) (namely $\theta= \pm \frac{\pi}{2}$ ). That is why this motion is impossible to achieve for the car modeled in the chained form (6) with $N_{c}=3$. The same problem was reported in [20] using the nilpotent approximation of the unicycle. Nevertheless, it should be noted that any motion along $\mathfrak{g}_{2} \backslash \mathfrak{g}_{1}$ (along $X_{3}=\left[X_{1}, X_{2}\right]=(0,0,-1,0)$ ), i.e. a pure rotation around the location of the rear wheels, can be achieved with $N_{c}=3$, whatever the coordinate system the car-like vehicle is written in.

\section{NMPC DESIGN FOR STATE FEEDBACK CONTROL OF CHAINED SYSTEMS}

The NMPC framework is now described in detail.

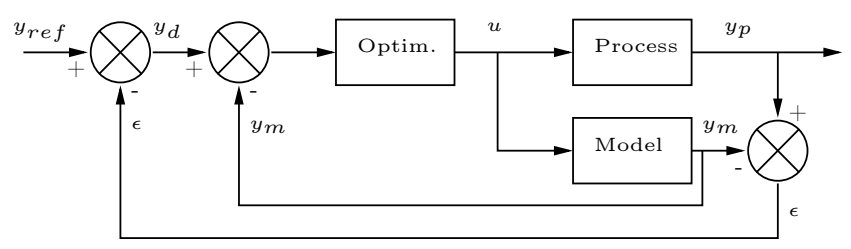

Fig. 1. Control Structure

1) The Internal Model Control (IMC) structure (see Fig. 1 ) is chosen to take into account modeling errors and disturbances [21]. The tracking of the reference trajectory $y_{r e f}$ by the process output $y_{p}$ is equivalent to the tracking of the desired trajectory $y_{d}$ by the model output $y_{m}$. At time $t=k T_{e}$ where the sampling period is $T_{e}=1 \mathrm{~s}$, the desired trajectory is defined by $y_{d}(k)=y_{\text {ref }}(k)-\varepsilon(k)$. The signal error $\varepsilon(k)=y_{p}(k)-y_{m}(k)$ includes modeling errors and measurement noises. The error is assumed to be constant over the prediction horizon but is updated at each new measurement. 


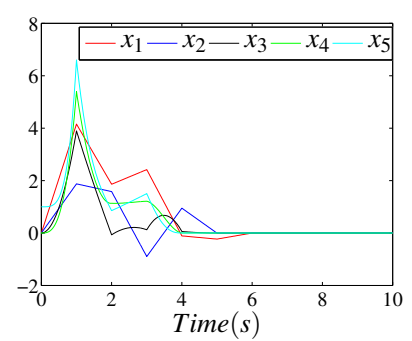

(a) Simulation 1: State variables.

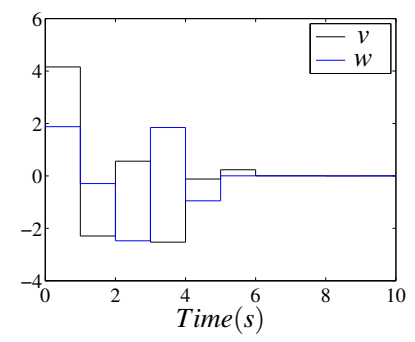

(e) Simulation 1: Control inputs.

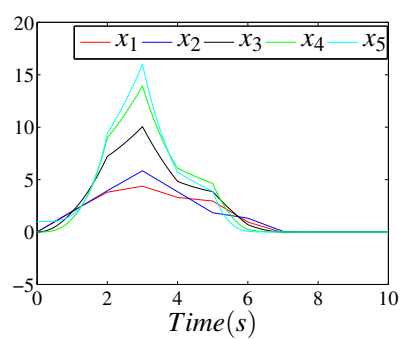

(b) Simulation 2: State variables.

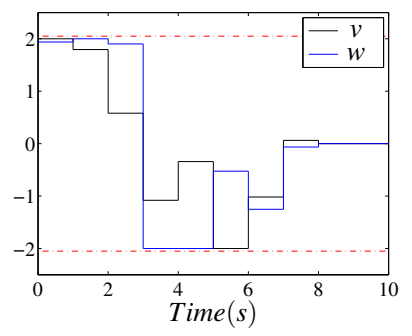

(f) Simulation 2: Control inputs.

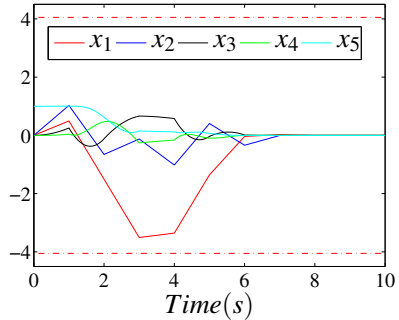

(c) Simulation 3: State variables.

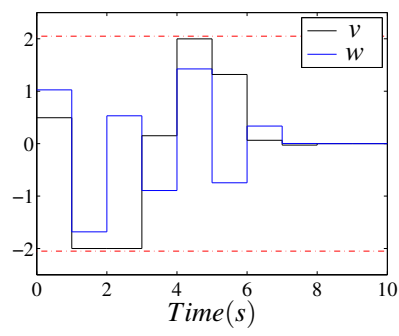

(g) Simulation 3: Control inputs.

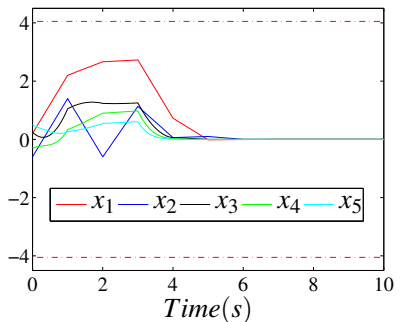

(d) Simulation 4: State variables.

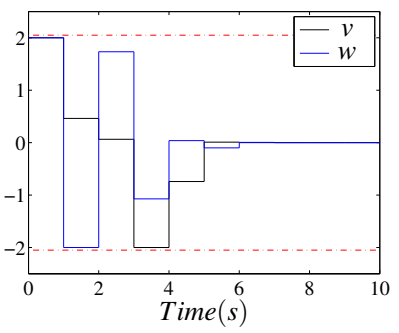

(h) Simulation 4: Control inputs.

Fig. 2. States and control variables of the chained system. Simulation 1: without constraints. Simulation 2: with control constraints. Simulations 3 and 4: with state and control constraints.

2) The model has to predict the process behavior over the prediction horizon.

$$
\left\{\begin{array}{l}
\dot{x}(t)=X_{1}(x(t)) v(t)+X_{2} w(t), x(0)=x_{0} \\
y_{m}(k+j \mid k)=x\left((k+j) T_{e}\right)
\end{array}\right.
$$

The variables $x \in \mathbb{R}^{n},(v, w) \in \mathbb{R}^{2}$ and $y_{m} \in \mathbb{R}^{n}$ are respectively the state, the input and the output of the model. The output is sampled at each $T_{e}$ and the computed input is kept constant over a sampling period: $\forall j \in\left[1 ; N_{p}\right]$,

$$
\begin{aligned}
& v(t)=v(k+j-1 \mid k) \text { for }(k+j-1) T_{e}<t<(k+j) T_{e}, \\
& w(t)=w(k+j-1 \mid k) \text { for }(k+j-1) T_{e}<t<(k+j) T_{e} .
\end{aligned}
$$

The model output $y_{m}(k)$, needed for the prediction, is initialized with the updated measure of the process output $y(k)$ that guarantees an implicit feedback of the IMC structure.

3) The quadratic function $L(1)$ is defined as:

$$
L=\left[y_{d(k+j k)}-y_{m(k+j k)}\right]^{T} Q_{(j)}\left[y_{d(k+j k)}-y_{m(k+j k)}\right]
$$

where $Q_{(j)}$ is a symmetric definite positive matrix.

4) The prediction horizon $N_{p}$ should satisfy a compromise between closed-loop stability (long horizon) and numerical feasibility in terms of computational time required (short horizon). If $N_{p}$ tends to infinity, the control problem becomes an optimal control known to ensure closedloop stability [11].

5) The control horizon $N_{c}$ plays a crucial role by proposing $N_{c}$ different controls at time $k$ comparable to a manoeuvre. According to Proposition 1, the control horizon will be chosen equal to or greater than the degree of nonholonomy of the chained system: $N_{c} \geq p+1$.

6) The time-varying matrix $Q(j)$ weights the tracking error at each iteration more and more over the prediction horizon. It reinforces the role of the terminal constraint. Giving a heavy weight at the end of $N_{p}$, which corresponds to the final objective, makes the NMPC problem (2) tend to the theoretical Problem 1. The time variation of the weighted matrix $Q(j)$ is given by:

$$
Q(j)=\alpha Q(j-1), \text { with } \alpha \geq 1 .
$$

7) The resolution method: a sequential quadratic programming (SQP) is used (function fminunc from Matlab optimization toolbox) to solve the optimization problem (2) with (11)-(14). We have deliberately chosen a standard algorithm to show that the efficiency of the NMPC for full-state control is due to the control horizon and does not depend on the optimization algorithm.

\section{SIMULATIONS}

We first illustrate the role of $N_{c}$ as a necessary condition for the state feedback of chained systems without constraints. We consider a $(2,5)$ chained system, described by the following vector fields:

$$
X_{1}(x)=\left(1,0, x_{2}, x_{3}, x_{4}\right), \quad X_{2}=(0,1,0,0,0) .
$$

The degree of nonholonomy is given by $p=n-1=4$. The prediction horizon and the control horizon were respectively set to $N_{p}=10$ and $N_{c}=5$. The time variation of $Q(j)$ is given by (14) with $\alpha=5$ and $Q(1)=I$.

We study the particular case where the initial state is $(0,0,0,0,1)$ and the desired one is $(0,0,0,0,0)$, corresponding to the difficult motion that requires a lateral displacement. With $N_{c}<5$, the final position is not reached. With $N_{c}=5$, in accordance with Proposition 1, the control strategy is able to determine a control sequence such that the fullstate converges to the desired state (see Fig. 2(a) and 2(e)). Constraints handling is one of the advantages of NMPC 
design. In simulation 2 , the control inputs of the $(2,5)$ chained system are constrained to \pm 2 . We can notice that the convergence is still obtained while the control constraints are satisfied (see Fig. 2(b) and 2(f)). The time of convergence is a bit longer and the amplitude of the state evolution is also greater. In Fig. 2(c) and 2(g), the state variables are also constrained to \pm 4 . The control objective is always achieved. From a different initial state $(0.2,-0.6,0.25,-0.3,0.5)$, the control strategy finds a control sequence satisfying the same constraints and the task (see Fig. 2(d) and 2(h)).

Remark 4 For all the presented simulations performed on a PC intel Core 2 duo, $3.06 \mathrm{GHz}$ under Matlab, the computational time required to solve the optimization problem was about 2 seconds. This computational time is composed of the Matlab function call, the differential equations and the optimization solving. The over-all computational time can be greatly reduced by using a discrete-time model of the mobile robot (simulations have already been performed and required $50 \mathrm{~ms}$ ) and by using a more efficient minimization algorithm. It is worth mentioning that the computational load relative to the usual choice of $N_{c}=N_{p}$ is divided by 3 approximately by using the appropriate choice of $N_{c}=p+1$.

\section{CONCLUSION}

An NMPC strategy has been applied to the state feedback control of chained systems. From a theoretical point of view, we have established a necessary condition on the length of the control horizon to ensure the NMPC feasibility. To achieve this, we have combined tools from differential geometry with NMPC approach. The link to the degree of nonholonomy provides a useful lower bound for the control horizon. Based on differential geometry, the proof of the necessary condition is given whatever the dimension of the chained system. From a practical point of view, the appropriate choice of the control horizon makes it possible to optimize the computational time. The theoretical results were illustrated on a car-like vehicle with one trailer, modeled by a chained system.

\section{APPENDIX \\ PROOF OF PROPOSITION 1}

Proof: The proof consists in four steps. The first step aims at reformulating the Problem 1 using the properties of the $(2, n)$ chained systems. The second and third steps are devoted to the study of cases where $s(\tilde{v})=e^{X}(0), X \in$ $\mathfrak{g} \backslash \mathfrak{g}_{1}$ and $s(\tilde{\boldsymbol{v}})=e^{X}(0), X \in \mathfrak{g} \backslash \mathfrak{g}_{N_{c}-2}$, that is motions in the direction of vector fields of increasing length, and thus of increasing difficulty. The last step concludes the proof.

1) First, we can make the Problem 1 more explicit for the $(2, n)$ chained system. The nilpotent algebra generated by the vector fields of (6) underlies the following group operation on the Lie group $N=\mathbb{R}^{n}$ :

$$
x y=\exp \left(A y_{1}\right) x+y
$$

where $A$ is the square matrix of dimension $n$ which nonzero entries are only $a_{i+1, i}=1, \forall i \geq 2$.
Since the vector fields of system (6) are left-invariant with respect to the group operation given by (A.16), (10) can be expressed as a product:

$$
s: \tilde{v} \mapsto x_{f}=x_{0} e^{Y_{1}}(0) e^{Y_{2}}(0) \cdots e^{Y_{N_{c}}}(0)
$$

with 0 denoting the identity element of $N=\mathbb{R}^{n}$. As the final configuration $x_{f}$ is any element of $N$, the initial condition can be set to $x_{0}=0$ without loss of generality. Thus (A.17) becomes $s: \tilde{v} \mapsto x_{f}=x(1) x(2) \cdots x\left(N_{c}\right)$, where $x(j)=e^{Y_{j}}(0)$ is the solution of equation (6) for the $j^{\text {th }}$ control input pair $\left(v_{j}, w_{j}\right)$ and initial condition 0 . Let $T_{e}=1 s$ so as not to burden notations, then using direct integration of (6), we get:

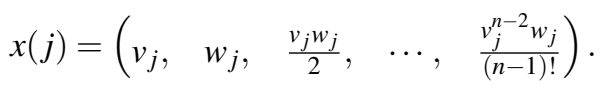

Using the group operation (A.16) on $\mathbb{R}^{n}$, the solution to a succession of $N_{c}$ iterations is thus given by:

$$
s(\tilde{\mathrm{v}})=\prod_{j=1}^{N_{c}} x(j)=\sum_{j=1}^{N_{c}-1} \exp \left(A \sum_{i=j+1}^{N_{c}} v_{i}\right) x(j)+x\left(N_{c}\right)
$$

Using (A.18) in the previous equation leads to:

$$
s(\tilde{v})=\left(\begin{array}{cc}
1 \cdots 1 & 0 \cdots 0 \\
0 \cdots 0 & 1 \cdots 1 \\
0_{n-2, N_{c}} & B(\tilde{v})
\end{array}\right) \tilde{v}
$$

with matrix $B$ elements $b_{i, N_{c}}=v_{N_{c}}^{i} /(i+1)$ ! and

$$
b_{i, j}=\sum_{r=0}^{i} \frac{\left(\sum_{q=j+1}^{N_{c}} v_{q}\right)^{i-r} v_{j}^{r}}{(i-r) !(r+1) !}, \quad \forall j \leq N_{c}-1 .
$$

2) If $s(\tilde{v})=e^{X}(0), X \in \mathfrak{g} \backslash \mathfrak{g}_{1}$, i.e. the shift's two first components are null while the others are not, (A.19) gives:

$$
\left(s_{3}, \ldots, s_{n}\right)(\tilde{v})=\bar{B}(\tilde{v}) \tilde{w},
$$

with, $\forall i \leq n-2, \forall j \leq N_{c}-1$, coefficients of $\bar{B}$ given by:

$$
\bar{b}_{i, j}=\frac{(-1)^{i+1}\left[\left(\sum_{r=1}^{N_{c}-1} v_{r}\right)^{i}-\sum_{q=0}^{i}\left(\begin{array}{c}
i+1 \\
q
\end{array}\right) v_{j}^{i-q}\left(\sum_{r=1}^{j-1} v_{r}\right)^{q}\right]}{(i+1) !} \text {. }
$$

Using (A.22), we get $\sum_{j=1}^{N_{c}-1} v_{j} \bar{b}_{i, j}=0, \forall i$, and (A.21) gives:

$$
\left(s_{3}, \ldots, s_{n}\right)(\tilde{v})=D(\tilde{v}) \lambda,
$$

where $D$ denotes the $N_{c}-2$ first columns of $\bar{B}$, and $\lambda=\left(\lambda_{1}, \ldots, \lambda_{N_{c}-2}\right)$ with $\lambda_{j}=w_{j}-v_{j} w_{N_{c}-1} / v_{N_{c}-1}$.

3) We will now show that, with a control sequence of length $N_{c}$, having $s(\tilde{v})=e^{X}(0)$ with $X \in \mathfrak{g} \backslash \mathfrak{g}_{N_{c}-2}$ implies that $s_{N_{c}}(\cdot)$ divides $s_{N_{c}+1}(\cdot), \cdots, s_{n}(\cdot)$.

Let $s(\tilde{v})=e^{X}(0)$ with $X \in \mathfrak{g} \backslash \mathfrak{g}_{N_{c}-2}$. We have $s_{i}(\cdot)=0$, $\forall i \leq N_{c}-1$ and (A.23) can be decomposed as:

$$
\left(\begin{array}{c}
0_{1, N_{c}-3} \\
s_{N_{c}} \\
\vdots \\
s_{n}
\end{array}\right)(\tilde{v})=\left(\begin{array}{cc}
C & E \\
\bar{b}_{N_{c}, 1} & R_{N_{c}} \\
\vdots & \vdots \\
\bar{b}_{n, 1} & R_{n}
\end{array}\right)(\tilde{v}) \lambda
$$


where the matrix $D$ is partitioned in blocks $C, R_{N_{c}+q}$ and $E$ respectively denote $\left(N_{c}-3\right)$ column and raw vectors and a $\left(N_{c}-3\right) \times\left(N_{c}-3\right)$ matrix. Using the Schur complements for $s_{N_{c}+q}(\cdot), \forall q=0, \cdots, n-N_{c}$, we get:

$$
s_{N_{c}+q}(\cdot)=\left(\bar{b}_{N_{c}+q, 1}-R_{N_{c}+q} E^{-1} C\right) \lambda_{1} .
$$

With the block matrix $F_{N_{c}+q}$ defined by:

$$
F_{N_{c}+q}=\left(\begin{array}{cc}
C & E \\
\bar{b}_{N_{c}+q, 1} & R_{N_{c}+q}
\end{array}\right),
$$

the equation (A.25) leads to:

$$
s_{N_{c}+q}=\frac{\operatorname{det} F_{N_{c}+q}}{(-1)^{N_{c}-3} \operatorname{det} E} \lambda_{1} .
$$

The determinants in the latter equation are determined using a LU decomposition with a unit triangular matrix $L$. Let $F_{N_{c}+q}=L_{N_{c}+q} U_{N_{c}+q}$, then the diagonal matrix $U_{n_{c}+q}$ coefficients are given using (A.22):

$$
U_{i, i}= \begin{cases}\frac{(-1)^{i+1}}{(i+1) !}\left(\sum_{j=i+1}^{N_{c}-1} v_{j}\right) \prod_{j=1}^{i-1}\left(\sum_{r=j}^{i} v_{r}\right), & \text { if } i \leq N_{c}-3 \\ \frac{(-1)^{N_{c}-1}}{\left(N_{c}-1\right) !} v_{N_{c}-1} \prod_{j=1}^{N_{c}-3}\left(\sum_{r=j}^{N_{c}-2} v_{r}\right) P_{N_{c}, q}, & \text { if } i=N_{c}-2\end{cases}
$$

with $P_{N_{c}, q}$ given by:

$$
\begin{aligned}
P_{N_{c}, q}= & \frac{(-1)^{q}\left(N_{c}-1\right) !}{\left(N_{c}+q-1\right) !} \sum_{|I|=q}\left(\begin{array}{c}
N_{c}+q-2 \\
I_{1}
\end{array}\right)\left(\begin{array}{c}
N_{c}+q-3-I_{1} \\
I_{2}
\end{array}\right) \\
& \cdots\left(\begin{array}{c}
q+1-\sum_{j=1}^{N_{c}-3} I_{j} \\
I_{N_{c}-2}
\end{array}\right) v_{1}^{I_{1}} \cdots v_{N_{c}-1}^{I_{N_{c}-1}}
\end{aligned}
$$

where the sum is done for multiindex $I=\left(I_{1}, \cdots, I_{N_{c}-1}\right)$ such that $|I|=q$, i.e. for every $I_{j}$ such that $\sum_{j=1}^{N_{c}-1} I_{j}=q$. Combining (A.28) and (A.29), we finally obtain:

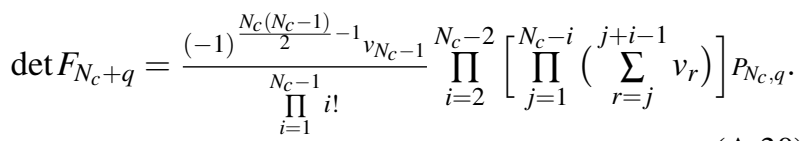

In the same way, we obtain $\operatorname{det} E$ :

$$
\begin{aligned}
\operatorname{det} E= & \frac{(-1)^{\frac{N_{c}\left(N_{c}-1\right)}{2}+1}}{N_{c}-2}\left\{\prod_{i=2}^{N_{i=1}^{N_{c}-3}}\left[\prod_{j=2}^{N_{c}-1-i}\left(\sum_{r=j}^{j+i-1} v_{r}\right)\right]\right\} \\
& \left\{\sum_{i=0}^{N_{c}-3}\left[\prod_{j=1}^{i}\left(\sum_{r=1}^{j} v_{r}\right) \prod_{j=1}^{N_{c}-3-i}\left(-\sum_{r=1}^{j} v_{N_{c}-r}\right)\right]\right\} .
\end{aligned}
$$

Using (A.30) and (A.31), (A.27) is simplified as:

$$
s_{N_{c}+q}(\cdot)=\frac{(-1)^{N_{c}-1} v_{N_{c}-1} \prod_{i=2}^{N_{c}-2}\left[\left(\sum_{j=1}^{i} v_{j}\right)\left(\sum_{j=1}^{i} v_{N_{c}-j}\right)\right] P_{N_{c}, q} \lambda_{1}}{\left(N_{c}-1\right) ! \sum_{i=0}^{N_{c}-3}\left[\prod_{j=1}^{i}\left(\sum_{r=1}^{j} v_{r}\right) \prod_{j=1}^{N_{c}-3-i}\left(-\sum_{r=1}^{j} v_{N_{c}-r}\right)\right]} .
$$

As $P_{N_{c}, 0}=1$, the equation (A.32) implies that

$$
s_{N_{c}+q}(\tilde{v})=s_{N_{c}}(\tilde{v}) P_{N_{c}, q}(\tilde{v}), \forall q=1, \cdots,\left(n-N_{c}\right) .
$$

Consequently, $s(\tilde{v})=e^{X}(0)$ with $X \in \mathfrak{g} \backslash \mathfrak{g}_{N_{c}-1}$ will involve that $s(\tilde{v})=0$. Hence, $s$ is not surjective for $N_{c} \leq p=n-1$.

4) For $N_{c}=p+1=n, s(\tilde{v})=e^{X}(0)$ with $X \in \mathfrak{g} \backslash \mathfrak{g}_{p-1}$ will have solutions provided that the numerator in (A.32) is not null, that is if $v_{N_{c}-1} \neq 0$ and no sum of strictly less than $N_{c}-2$ successive controls $v_{i}$ is null. As a result, $s$ is surjective for $N_{c}=p+1$.

\section{ACKNOWLEDGMENT}

The authors are very grateful to Pascal Morin for his helpful advices.

\section{REFERENCES}

[1] O. J. Sørdalen, "Conversion of the kinematics of a car with $\mathrm{n}$ trailers into a chained form," IEEE Trans. on Automatic Control, vol. 40, pp. $35-49,1993$.

[2] P. Morin and C. Samson, "Trajectory tracking for non-holonomic vehicles : overview and case study," IEEE Workshop on Robot Motion Control, pp. 109-153, 2004.

[3] — Handbook of Robotics. Springer, 2008, ch. Motion Control of Wheeled Mobile Robots.

[4] R. Brockett, "Asymptotic stability and feedback stabilization," in Differential Geometric Control Theory. Birkauser, 1983.

[5] C. Samson, "Velocity and torque feedback control of a nonholonomic cart," Int. Workshop in Adaptative and Nonlinear Control: Issues in Robotics, 1990, also in LNCIS, Vol. 162, Springer Verlag, 1991.

[6] J.-B. Pomet, "Explicit design of time-varying stabilizing control laws for a class of controllable systems without drift," Systems \& Control Letters, vol. 18, pp. 467-473, 1992.

[7] R. M'Closkey and R. Murray, "Exponential stabilization of driftless nonlinear control systems using homogeneous feedback," IEEE Trans. on Automatic Control, vol. 42, pp. 614-628, 1997.

[8] C. Canudas de Wit and O. J. Sørdalen, "Exponential stabilization of mobile robots with nonholonomic constraints," IEEE Trans. on Automatic Control, vol. 37, no. 11, pp. 1791-1797, 1992.

[9] M. K. Bennani and P. Rouchon, "Robust stabilization of flat and chained systems," in European Control Conference, 1995, pp. 2642 2646.

[10] P. Morin and C. Samson, "Control of nonholonomic mobile robots based on the transverse function approach," IEEE Trans on Robotics, vol. 25 , no. 5, pp. 1058-1073, 2009

[11] E. Camacho and C. Bordons, "Nonlinear model predictive control: An introductory review," in Assessment and Future Directions of Nonlinear Model Predictive Control. Springer Berlin / Heidelberg, 2007, vol. 358, pp. 1-16.

[12] H. van Essen and H. Nijmeijer, "Nonlinear model predictive control for constrained mobile robots," European Control Conference, 2001.

[13] M. Alamir and N. Marchand, "Constrained minimum-time-oriented feedback control for the stabilization of nonholonomic systems in chained form," Journal of Optimization Theory and Applications, pp. 229-244, 2003.

[14] G. Grimm, M. Messina, S. Tuna, and A. Teel, "Model predictive control: for want of a local control lyapunov function, all is not lost," IEEE Trans. on Automatic Control, vol. 5, pp. 229-244, 2005.

[15] F. Warner, Foundations of differential manifolds and Lie groups. Springer Verlag, 1983.

[16] H. Nijmeijer and A. Van der Schaft, Nonlinear Dynamical Control Systems. Springer Verlag, 1991.

[17] C. C. de Wit and C. Samson, "Nonlinear feedback control," in Theory of robot control. Springer Verlag, 1996.

[18] V. Gershkovich and A.Vershik, "Nonholonomic manifolds and nilpotent analysis," Journal of Geometry and Physics, vol. 5, no. 3, pp. 407-452, 1988

[19] H. Hermes, A. Lundell, and D. Sullivan, "Nilpotent bases for distributions and control systems," Journal of Differential Equations, vol. 55 no. 3, pp. 385-400, 1984.

[20] G. Lafferriere, "A general strategy for computing steering controls of systems without drift," in IEEE Conference on Decision and Control, 1991, pp. 1115-1120.

[21] M. Morari and E. Zafiriou, "Robust control.” Dunod, 1983. 